\title{
Forward-scattering of meteors: a digital way
}

\author{
Saša Nedeljković ${ }^{1}$ and Vjera Miović ${ }^{2}$ \\ University of Toronto, 60 St. George Street, Toronto, M5S 1A7, Canada \\ ${ }^{1}$ email: sasa@physics.utoronto.ca \\ ${ }^{2}$ email: miovic@physics.utoronto.ca
}

\begin{abstract}
The new cutting edge technologies have opened the possibility of using digital spectrometers to probe the sky. With the sampling rate of $\sim 400 \mathrm{Msamples/s}$ and the capability to digitally process data in real-time it is possible to get wideband spectra covering the frequencies in the range $0-200 \mathrm{MHz}$ with the resolution of a few $\mathrm{kHz}$. This presentation will show how such a spectrometer can be applied to the forward-scattering method of meteor detection.
\end{abstract}

Keywords. Meteors, atmospheric effects, instrumentation: spectrographs, radio lines: general

\section{Introduction}

Advances in high speed digital electronics and data acquisition have enabled the development of the new radio frequency spectrometers with the capability of exploring exciting astrophysical questions. Modern analog/digital (A/D) converters are capable of transforming the analog signals of up to $1 \mathrm{GHz}$ into a digital form. At the same time the processing power of inexpensive computers is becoming sufficient for the real time data analysis.

A digital spectrometer can be described as a system composed of an antenna, an amplifier, AD converter and a computer. At the University of Toronto, we are currently building such an instrument, known as TREX (21 cm Reionization EXperiment) to both probe the Epoch of Reionization and to characterise the micrometeorite arrival statistics.

TREX is designed to work with frequencies up to $200 \mathrm{MHz}$. To achieve $200 \mathrm{MHz}$ Nyquist frequency we need to sample data using $400 \mathrm{MHz}$ A/D converter. This exceeds the transfer rate of all commercially available A/D converters, which typically are only able to transfer a few $\mathrm{MB} / \mathrm{sec}$, leading to very inefficient data acquisition. An ongoing project to develop a faster A/D converter and acquisition system is under way at the Physics Electronics Resource Centre at the University of Toronto. 64-bit PCI-X bus will be used to transfer all data to the computer's memory. Such a design will permit streaming of all data to the computer's memory in real time. After the data is received in the main memory, fast Fourier transform (FFT) needs to be performed to obtain the power spectrum. FFT calculation is a computationally very intense task. $N$-point FFT uses $\frac{1}{2} N \log _{2} N$ operations. Even for the fastest personal computers today, this is a challenging computational task. In the next section we will discuss a possible way of speeding up the FFT calculation by using Intel's SIMD (Single Instruction Multiple Data) technology.

The meteor detection via forward-scattering needs a properly chosen location for the receiver. The forward-scattering technique uses transmitters which are at a different location than the receiver. The transmitter-receiver system must not be in the line-ofsight. In section 3 we discuss how to find a proper place for the receiver. 


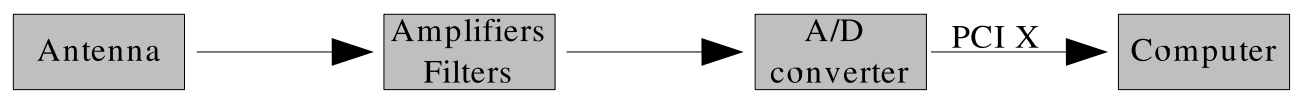

Figure 1. TREX schematics

\section{Digital Spectrometer}

\subsection{Power Spectrum}

Obtaining the data for a single antenna system is quite simple: from the input signal the power spectrum must be calculated. This is the most time costly operation, so a carefully chosen algorithm is crucial. Two well known FFT packages are FFTW (Fastest Fourier Transform in the West) and Intel's IPP (Integrated Performace Primitives). These algorithms are optimised for working with the floating-point numbers. For the purpose of our digital spectrometer we will use integer instead of the floating-point arithmetics to additionally speed up the calculations. Intel's processor streaming SIMD extensions can be used to speed up the FFT algorithm by adapting it to the specific hardware such as Pentium 4.

\subsection{FFT code}

New Intel's microprocessors such as Pentium 4 and Itanium have implemented a set of instructions for calculations with multiple data in a single instruction - SIMD processing. An excellent reference for the interested reader is Intel $\mathrm{C}++$ Compiler for Linux Systems User's Guide (2003). Primarily designed for speeding up multimedia applications, these instructions can be used for speeding up almost any programming task. Implementation is done trough extensions to previously defined/implemented instructions. From the programmer's point of view, the usage of SIMD can be implemented using the inlined assembly code, or even simpler, by using Intel's C++ API extension sets, better known as intrinsics. Intrinsics are the special coding extensions that allow using the syntax of $\mathrm{C}$ function calls and $\mathrm{C}$ variables instead of the hardware registers. Using these intrinsics frees programmers from having to program in the assembly language and to manage registers manually. The compiler optimization is automated so the instructions are scheduled to maximise the running speed.

SIMD extensions use eight 128-bit registers capable of processing the data elements in parallel. Since each of the registers can store more than one data element, the processor can operate with more data simultaneously.

An algorithm is written to use SIMD2 extensively with 16-bit data (short). Each 128bit SIMD register can hold and simultaneously operate with eight 16-bit integer values. We are computing eight FFTs simultaneously by packing eight 16-bit data in one 128-bit SIMD type. Which means that our algorithm performs eight FFTs at the cost of one.

We have tested the FFT performance of this new algorithm and compared the results with the FFTW code working with 64-bit data and 32-bit data. The results are presented on Figure 2.

Our algorithm shows a faster execution time by a factor of two. The drop in speed at the array size of $2^{14}$ is due to the processor cache memory. FFTW is also affected by cache limitation but with the larger array sizes. We are initially using eight 16-bit data arrays compared to only one 32 or 64-bit data array. One might expect a greater difference in the execution speed knowing that 8 FFTs are being executed at the cost of one. However, FFTW also uses the SIMD technology to speed up the execution time. FFTW parallelises groups of instructions if possible. For example if there are two consecutive additions they can be done in parallel. Such an optimisation is limited by compiler/programmer ability to transfer the linear code into parallel. A good example of instructions which can not 


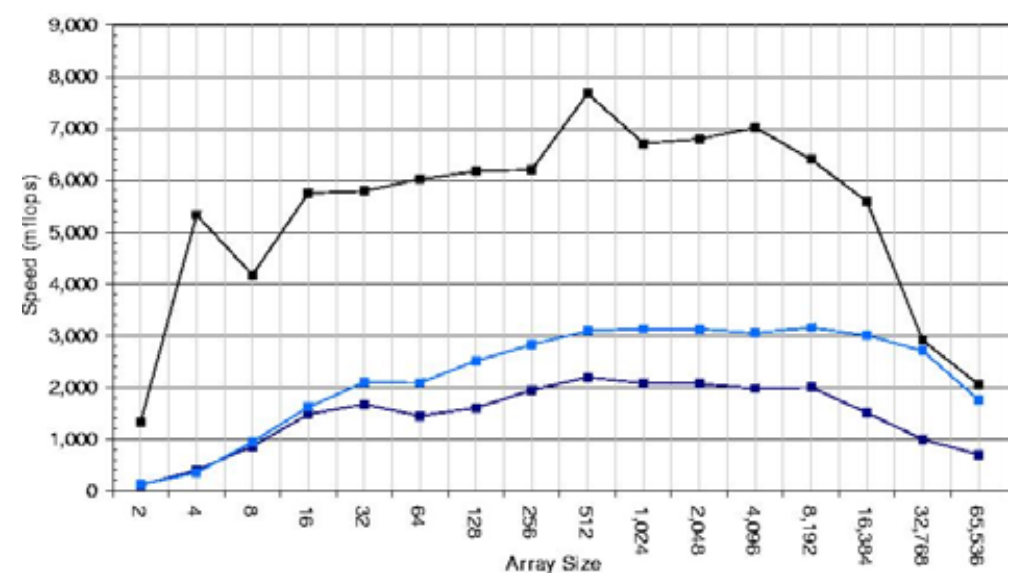

Figure 2. Speed comparison of SIMD2 optimised FFT code (top line) with the FFTW algorithm working with 32-bit floating point data (middle line) and 64-bit double floating point data (bottom line). The tests were performed on a $2.4 \mathrm{GHz}$ Intel Xeon machine.

be done in parallel is

$$
\begin{gathered}
x=3+5 \\
y=x+3,
\end{gathered}
$$

because the input of the second equation depends on the result of the first one.

There are two limitations to our code. The first one is data overflow caused by 2 byte data size limitation. If the result of any operation is longer than 2 bytes, the overflow will be generated. There is no cure for this, since the overflow testing would slow down the algorithm by a factor of $\sim 10$. The second one is due to the arithmetic data shifting. Since the division in the set of short numbers is not hardware defined, one has to use data shifting to perform this operation. To divide a binary number by 2 it is enough to arithmetically shift the binary number one place to the right. The shifting also carries the signum, so that for example, $1 / 2=0$, but $-1 / 2=-1$. It causes the error in the FFT result. However, despite the cost in the result precision, this algorithm can be used for the detection of individual spectral lines.

The comparison in precision is shown on Figure 3. At the two 16-bit sets in the Figure 3 it is easy to notice the "ghost" line at $155.9 \mathrm{MHz}$. The origin of this "ghost line" is the data overflow. At frequencies at about $70-80 \mathrm{MHz}$ in the bottom spectra there are ghost lines originating from the division error discussed above. Despite these two types of errors the spectra calculated using our algorithm can be used for scientific data analysis. These errors are "constant" in time if the spectra do not change. However, a change in spectra might affect the errors. In the case of spectral lines caused by meteor forward-scattering, the error is negligible.

\section{Where to observe from?}

Man-made interference can be the source of contamination of the astronomical signals. Radio signals of astronomical origin are by far weaker than the signals used by terrestrial communication systems. The interference can result in "bad data" and even in completely wrong scientific interpretation.

FM stands for "Frequency Modulated". The FM radio band goes from $88 \mathrm{MHz}$ up to $108 \mathrm{MHz}$. To resolve different stations the FM signals must be at least $200 \mathrm{kHz}$ apart. 

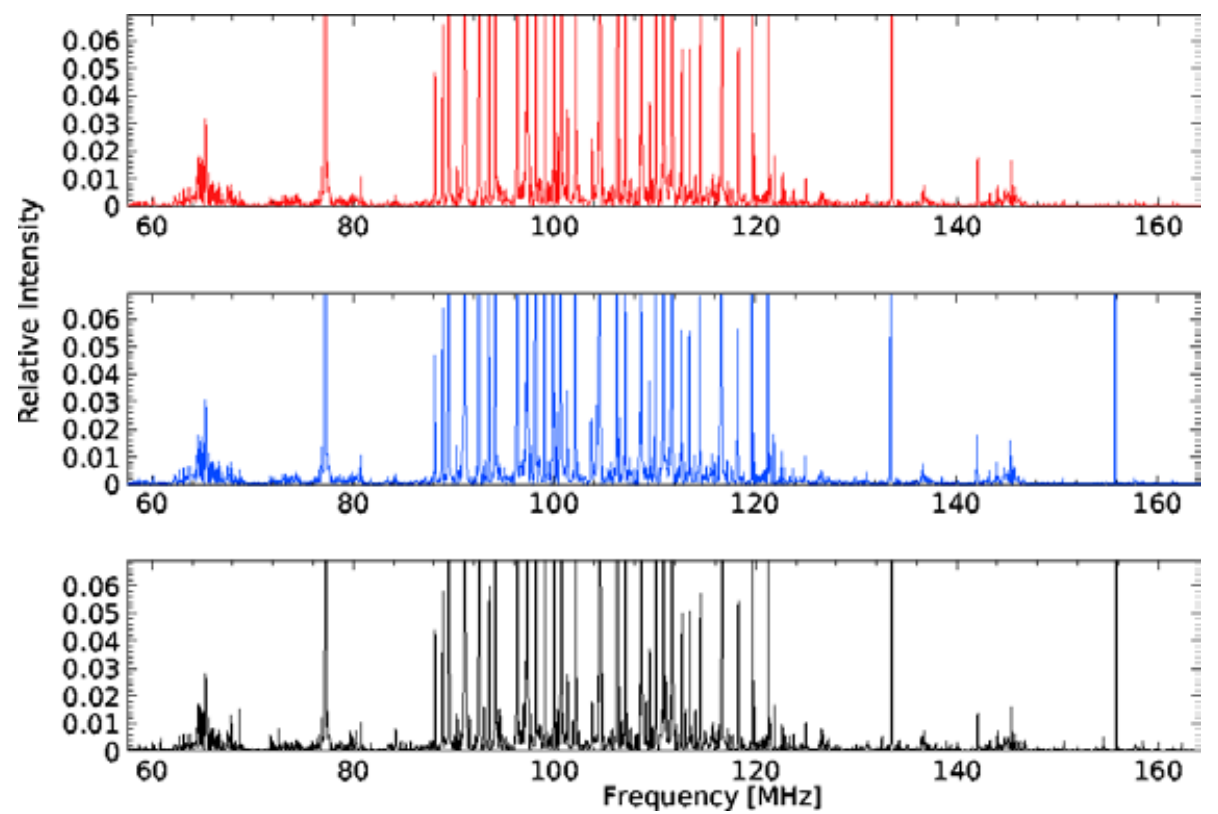

Figure 3. Comparison of spectra calculated from the same dataset using different data types: 32-bit floating point (top), 16-bit integer (middle) and 16-bit integer using SIMD2 instructions (bottom). For the two bottom spectra, FFT is done with 16-bit precision and the final power spectra are calculated as 32-bit integers. Data were obtained using a rectangular approximation of two arm conical spiral antenna at the Department of Physics, University of Toronto on July 28th, 2004.

Knowing that the total FM bandwidth is $20 \mathrm{MHz}$, there can not be more than 100 stations visible in the whole FM band.

There is no reflection from the Earth's ionosphere in the FM band, which means that the receiver must be in the line-of-sight of the transmitter in order to receive data. However, one should keep in mind that the signals can be reflected from mountains and buildings. The maximum distance one can receive the FM signal from depends on the curvature of the Earth, terrain configuration and the position of the receiver and the transmitter.

The maximum distance $l$ a receiver (at the sea level) can receive a line-of-sight signal from a transmitter at the altitude $h$ (above the sea level) is given by the expression:

$$
l=R \arctan \frac{\sqrt{h^{2}+2 R h}}{R},
$$

where $R=6380 \mathrm{~km}$ is the Earth's radius and $h$ is the height of the transmitter. To avoid interference coming from the line-of-sight terrestrial receivers at height $h$ one must be further than the distance $l$ away.

For example, the famous CN Tower is often stated as the tallest free-standing structure in the world. Located in Toronto, Canada, completed in 1976, the tower stands at 553 meters (including antennas). Using the equation above, the maximum distance at which we can hear the signal emitted from the top of the CN Tower is about $85 \mathrm{~km}$. 


\section{Meteor Detection}

When a meteoroid enters the region of the ionosphere in the altitude range of 80 120 kilometers, it produces a trail of free electrons due to collisions with air molecules. The high kinetic energy involved in the process determines the transformation of a solid body into a plasma, which can scatter radio waves and emit light (meteor). Signals produced by FM radio and TV stations, being reflected from a meteor trail, might be used to extract all vital information describing the meteor.

When the transmitter (radio or TV station) is located far enough from the receiver, so that direct contact is impossible due to the curvature of the Earth, the signal reflected from a meteor trail can be detected. Such technique is known as forward meteor scattering. Even by knowing all characteristics of the transmitter and the receiver beams, when the receiver operates only on one fixed frequency, the orbital elements of a meteor can not be resolved. One solution commonly used is to have one transmitter and more than one receiver in order to collect enough information so that the meteor parameters can be determined by comparing the data from different receivers looking at the same trail.

A technically simpler system is composed of only one receiver: 2ACSA (2-Arm Conical Spiral Antenna) optimised for $80-250 \mathrm{MHz}$ coupled with a digital spectrometer. Such a system with a resolution of a few $\mathrm{kHz}$ can resolve different terrestrial VHF signals. All non-field-of-view VHF sources can be used as transmitters for forward scattering method. By knowing the frequency, directivity and the power of transmitters, and keeping in mind that there is a large number of suited sources, it is possible to extract all meteor parameters by having only one receiver. 2ACSA is an excellent choice for the receiver antenna because of its frequency independent characteristics.

The detected meteors could be coming from comet debris within the solar system, or they could be the micron size extrasolar dust particles. The proper method of detection could distinguish between solar and extra solar origin of meteoritic grains, once the orbital elements of the particles are known. The duration of the trail can give information about mass. Knowing the velocity of entry and height from the observations one can get the orbital elements of the meteor. This detection can yield valuable information for astronomy.

If electrons in plasma are offset from the equilibrium position, they execute simple harmonic motion. The equation of motion for the electrons gives a characteristic angular frequency of oscillation:

$$
\omega_{p}=\sqrt{\frac{n_{e} e^{2}}{\epsilon_{0} m_{e}}},
$$

where $n_{e}$ and $m_{e}$ are electron number density and mass, respectively. Since electrons move faster than ions, they mainly contribute to the plasma frequency, which is the collective characteristic of the plasma and is referred to as the Langmuir frequency.

There are two recognisable classes of meteors based on their Langmuir frequency: underdense and overdense. In underdense meteors, the electron density is small enough to allow the radio wave to propagate through the meteor and each individual electron scatters the incident electromagnetic wave. In the overdense meteor, the incident wave is totally reflected, and a simple model can be used in which the meteor trail is treated as a cylinder. In the overdense scatter, the Langmuir frequency is lower than that of the radio wave. In our observations, we can distinguish between overdense and underdense meteors by observing the time evolution of the signal. 
Using the plasma physics approach, a simple model of an overdense meteor can lead to obtaining the approximate height of a meteor, as shown by Foschini (1999):

$$
h \approx \frac{L}{2}\left(\frac{1}{\tan \phi}-\frac{L}{4 R_{\oplus}}\right),
$$

where $L=2 R_{\oplus} \sin \alpha$, and the angles $\phi$ and $\alpha$ are the half angular distance between the receiver and the transmitter, from the meteor trail and the centre of the Earth, respectively.

\section{Conclusion}

The advances in digital electronics allow us to develop a digital spectrometer which can be used to probe the epoch of reionisation in the universe and to do meteor physics. We have shown how such a system can be used to characterise meteor trail orbits and heights. A new algorithm has been developed to perform FFT in order to obtain power spectra of forward-scatter detected meteors. It is implemented on the Intel Pentium 4 SIMD processors. Because it performs $8 \mathrm{FFT}$ calculations at once and works only with integers, it is significantly faster than the conventional algorithms, without the loss of performance for our purposes. The spectrometer developed at the University of Toronto digitally processes data in real-time producing spectra in the range of frequencies covering up to $200 \mathrm{MHz}$.

\section{Acknowledgements}

The authors would like to thank Ue-Li Pen, Barth Netterfield and Anna Lubchenko for their involvement in this project.

\section{References}

Intel C++ Compiler for Linux Systems User's Guide, 2003, Intel Corporation

Foschini, L. 1999, Astron. Astrophys. 341, 634 